\title{
Wave Scattering by Small Undulation on the Porous Bottom of an Ocean in the Presence of Surface Tension
}

\author{
Srikumar Panda, ${ }^{1}$ Sudhanshu Shekhar Samantaray, ${ }^{2}$ and S. C. Martha ${ }^{1}$ \\ ${ }^{1}$ Department of Mathematics, Indian Institute of Technology Ropar, Nangal Road, Rupnagar 140 001, India
}

${ }^{2}$ Department of Mathematics, Utkal University, Vanivihar, Bhubaneswar 751 004, India

Correspondence should be addressed to S. C. Martha; scmartha@gmail.com

Received 30 April 2012; Accepted 26 June 2012

Academic Editors: C. Grenz and J. L. Zhou

Copyright (C) 2013 Srikumar Panda et al. This is an open access article distributed under the Creative Commons Attribution License, which permits unrestricted use, distribution, and reproduction in any medium, provided the original work is properly cited.

\begin{abstract}
The scattering of incident surface water waves due to small bottom undulation on the porous bed of a laterally unbounded ocean in the presence of surface tension at the free surface is investigated within the framework of two-dimensional linearized water wave theory. Perturbation analysis in conjunction with the Fourier transform technique is employed to derive the first-order reflection and transmission coefficients in terms of integrals involving the shape function $c(x)$ representing the bottom undulation. One special type of bottom topography is considered as an example and the related coefficients are determined in detail. These coefficients are presented in graphical forms. The theoretical observations are validated computationally. The results for the problem involving scattering of water waves by bottom deformations on an impermeable ocean bed are obtained as a particular case.
\end{abstract}

\section{Introduction}

The interaction problems of surface waves with a preexisting (fixed) pattern of undulation on an otherwise flat bed are important for their possible applications in the areas of coastal and marine engineering, and as such these are being studied for a long time. The problem of reflection of surface waves by patches of bottom undulations has received an increasing amount of attention as its mechanism is important in the development of shore-parallel bars.

The problems of water wave diffraction by undulating bottom topography of a sea bed have been considered by many scientists and several papers have been published in this direction (see Davies and Heathershaw [1], Mandal and Basu [2], Martha and Bora [3], Miles [4], and many others).

All the above works focused only on the wave motion over the impermeable bottom topography of sea bed in absence of surface tension at free surface. Nowadays, due to many interesting applications in the theory of water waves scattering, many researchers have turned their attention to the problems related to porous bed rather than an impermeable one. Mase and Takeba [5], Zhu [6], and Silva et al. [7] considered waterwave reflection and/or transmission problems where a porous medium was assumed to lie on a sea bed of varying quiescent depth. Martha et al. [8] focused on the problem involving wave scattering by small undulation on a porous bottom topography without considering the surface tension at the free surface.

Here, we investigate the scattering problem of surface water waves by small undulation on an porous ocean-bed of finite depth in the presence of surface tension at the free surface. The motion of the fluid below the porous ocean bed is not analyzed here and it is assumed that the fluid motions are such that the resulting boundary condition on the ocean bed as considered here holds good and depends on a known parameter $G$, called porosity parameter, in this analysis. Due to the small parameter $\varepsilon(\ll 1)$ being present in the representation of the small undulation of the bottom, the perturbation analysis can be employed. By this analysis, the velocity potential, the reflection, and transmission coefficients appearing in the governing boundary value problem (BVP) can be expanded as a power series involving this parameter $\varepsilon$. Equating the coefficients of identical powers of the parameter $\varepsilon$ from both sides of all equations appearing in the coupled BVP, a number of BVPs can be obtained. However, the BVP up to first order is considered here for 
its solutions as the BVP of higher order can be solved successively in principle. The solution of the zeroth order BVP is obvious. Using Fourier transform technique, the solution involving the velocity potential for the first order BVP is obtained. By comparing this solution with far-field behaviour of the velocity potential, the first-order reflection and transmission coefficients are derived in terms of integrals involving the shape function $c(x)$ representing the bottom undulation. These coefficients are computed numerically for a special form of bottom topography, namely, sinusoidal bottom, and depicted graphically. The results for problem involving scattering of water waves by bottom deformations on an impermeable ocean bed are obtained as a particular case.

\section{Problem Formulation}

A right-handed rectangular Cartesian coordinate system is considered in which $x$-axis is the position of the undisturbed free surface of the ocean and $y$-axis is positive vertically downward from the undisturbed free surface (see Figure 1). The ocean bed with small undulation, composed of porous material of specific type, is described by $y=h+\varepsilon c(x)$, where $c(x)$ is a bounded and continuous function, describing the undulation of the ocean bed and $c(x) \rightarrow 0$ as $|x| \rightarrow$ $\infty$ so that the ocean is of uniform finite depth $h$ far away from the undulation on either side; the nondimensional number $\varepsilon(\ll 1)$ is a small parameter giving a measure of the smallness of the undulation. It is also assumed that the fluid is incompressible and inviscid, and the motion is irrotational. The usual assumptions of linear water wave theory and the removal of the harmonic time independence $\exp (-i \sigma t)$ lead to the following governing equations for the time-independent complex-valued velocity potential function $\phi(x, y)$ :

$$
\begin{aligned}
& \frac{\partial^{2} \phi}{\partial x^{2}}+\frac{\partial^{2} \phi}{\partial y^{2}}=0, \quad \text { in } 0 \leq y \leq h+\varepsilon c(x),-\infty<x<\infty, \\
& \frac{\partial \phi}{\partial y}+K \phi+M \frac{\partial^{3} \phi}{\partial y^{3}}=0, \quad \text { on } y=0,-\infty<x<\infty, \\
& \frac{\partial \phi}{\partial n}-G \phi=0, \quad \text { on } y=h+\varepsilon c(x),-\infty<x<\infty,
\end{aligned}
$$

where $K=\sigma^{2} / g$ and $M=T / \rho g, \sigma$ is the angular frequency of the incoming water-wave train, $g$ is acceleration due to gravity, $T$ is the coefficient of surface tension at the free surface of the ocean, $\rho$ is the density of water, $\partial / \partial n$ denotes the normal derivative at a point $(x, y)$ on the bottom, and $G$ is the porous effect parameter corresponding to the ocean bed under consideration.

It is assumed that a train of progressive wave is incident to the bottom undulation from the direction of $x=-\infty$, then it is partially reflected by and partially transmitted over the

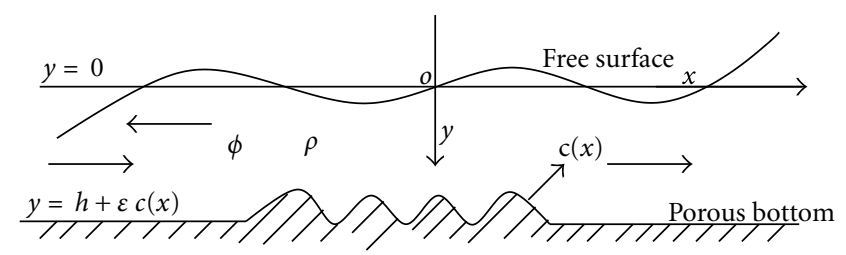

FIGURE 1: Definition sketch of wave propagation.

bottom undulation so that $\phi$ has the far-field behaviour given by

$$
\phi(x, y) \longrightarrow \begin{cases}\phi_{\mathrm{inc}}(x, y)+R \phi_{\mathrm{inc}}(-x, y), & x \longrightarrow-\infty, \\ T \phi_{\mathrm{inc}}(x, y), & x \longrightarrow \infty,\end{cases}
$$

where $R$ and $T$ represent, respectively, the unknown reflection and transmission coefficients which are to be determined here.

\section{Methodology and Results}

3.1. Perturbation Technique. Assuming $\varepsilon$ to be very small, the bottom boundary condition (3) can be expressed as

$$
\begin{aligned}
\frac{\partial \phi}{\partial y}-\varepsilon \frac{\partial}{\partial x}\left[c(x) \frac{\partial \phi}{\partial x}\right]-G\left[\phi+\varepsilon c(x) \frac{\partial \phi}{\partial y}\right]+ & O\left(\varepsilon^{2}\right)=0 \\
\text { on } y & =h .
\end{aligned}
$$

If there is no undulation at the bottom, the surface wave train propagates without any hindrance and there is total transmission. In view of this along with the approximate form of relation (5), $\phi, T, R$ can be expressed in terms of the small parameter $\varepsilon$ as follows:

$$
\begin{gathered}
\phi=\phi_{0}(x, y)+\varepsilon \phi_{1}(x, y)+O\left(\varepsilon^{2}\right), \\
T=1+\varepsilon T_{1}+O\left(\varepsilon^{2}\right), \\
R=\varepsilon R_{1}+O\left(\varepsilon^{2}\right) .
\end{gathered}
$$

Substituting the expansions (6a)-(6c) into (1)-(2) and (4)-(5), we find, after equating the coefficient of $\varepsilon^{0}$ and $\varepsilon$ from both the sides, that the functions $\phi_{0}(x, y)$ and $\phi_{1}(x, y)$, respectively, satisfy the following BVPs.

$B V P-I$. The function $\phi_{0}(x, y)$ satisfies

$$
\begin{gathered}
\frac{\partial^{2} \phi_{0}}{\partial x^{2}}+\frac{\partial^{2} \phi_{0}}{\partial y^{2}}=0, \quad \text { in } 0 \leq y \leq h,-\infty<x<\infty, \\
\frac{\partial \phi_{0}}{\partial y}+K \phi_{0}+M \frac{\partial^{3} \phi_{0}}{\partial y^{3}}=0, \quad \text { on } y=0, \\
\frac{\partial \phi_{0}}{\partial y}-G \phi_{0}=0, \quad \text { on } y=h .
\end{gathered}
$$


BVP-II. The function $\phi_{1}(x, y)$ satisfies

$$
\begin{aligned}
& \frac{\partial^{2} \phi_{1}}{\partial x^{2}}+\frac{\partial^{2} \phi_{1}}{\partial y^{2}}=0, \quad \text { in } 0 \leq y \leq h,-\infty<x<\infty \text {, } \\
& \frac{\partial \phi_{1}}{\partial y}+K \phi_{1}+M \frac{\partial^{3} \phi_{1}}{\partial y^{3}}=0, \quad \text { on } y=0, \\
& \frac{\partial \phi_{1}}{\partial y}-G \phi_{1}=\frac{d}{d x}\left[c(x) \frac{\partial \phi_{0}}{\partial x}\right]+G\left[c(x) \frac{\partial \phi_{0}}{\partial y}\right] \\
& \equiv V(x)(\text { say }) \text { on } y=h \text {, } \\
& \phi_{1}(x, y) \longrightarrow \begin{cases}R_{1} \phi_{\text {inc }}(-x, y), & x \longrightarrow-\infty, \\
T_{1} \phi_{\text {inc }}(x, y), & x \longrightarrow \infty .\end{cases}
\end{aligned}
$$

The solution of the BVP-I corresponds to the incident wave over finite depth $h$ and can be written as

$$
\phi_{0}(x, y)=\frac{1}{K}\left[\left(k_{0}+M k_{0}^{3}\right) \cosh k_{0} y-K \sinh k_{0} y\right] e^{i k_{0} x},
$$

where $k_{0}$, the wave number of the normal incident wave, is the unique positive root of the following dispersion relation:

$$
\left(K+G+M G k_{0}^{2}\right)-\left(k_{0}+\frac{G K}{k_{0}}+M k_{0}^{3}\right) \tanh k_{0} h=0 .
$$

Note that, $-k_{0}$ is the negative root of the dispersion relation (11).

3.2. Fourier Transform Technique. The solution of BVP-II is obtained by using Fourier transform technique. To solve this BVP, we now assume $k_{0}$ to have a small positive imaginary part so that $\phi_{1}$ decreases exponentially as $|x| \rightarrow \infty$. This ensures the existence of Fourier transform $\widehat{\phi}_{1}(\xi, y)$ of $\phi_{1}(x, y)$ with respect to $x$ and defined as follows:

$$
\widehat{\phi}_{1}(\xi, y)=\int_{-\infty}^{\infty} \phi_{1}(x, y) e^{-i \xi x} d x
$$

with the following inverse transform:

$$
\phi_{1}(x, y)=\frac{1}{2 \pi} \int_{-\infty}^{\infty} \widehat{\phi}_{1}(\xi, y) e^{i \xi x} d \xi
$$

Applying Fourier transform defined in relation (12), to (8a), $(8 \mathrm{~b})$ and $(8 \mathrm{c})$ and solving them, we obtain $\widehat{\phi}_{1}(\xi, y)$ as given by

$$
\begin{aligned}
& \widehat{\phi}_{1}(\xi, y) \\
& =\frac{\left[\left(\xi+M \xi^{3}\right) \cosh \xi y-K \sinh \xi y\right] \bar{V}(\xi)}{\left\{\xi\left(\xi+M \xi^{3}\right)+K G\right\} \sinh \xi h-\left\{K \xi+G\left(\xi+M \xi^{3}\right)\right\} \cosh \xi h},
\end{aligned}
$$

where $\bar{V}(\xi)$ is the fourier transform of $V(x)$.
Taking the inverse fourier transform, the solution for the first order velocity potential $\phi_{1}(x, y)$ can be written as

$$
\begin{aligned}
\phi_{1}(x, y)=\frac{1}{2 \pi} \int_{0}^{\infty} \frac{\left[\left(\xi+M \xi^{3}\right) \cosh \xi y-K \sinh \xi y\right]}{\xi \triangle(\xi)} \\
\times\left[\bar{V}(\xi) e^{i \xi x}+\bar{V}(-\xi) e^{-i \xi x}\right] d \xi
\end{aligned}
$$

where

$$
\begin{aligned}
& \triangle(\xi) \\
& =\left(\xi+\frac{G K}{\xi}+M \xi^{3}\right) \sinh \xi h-\left(K+G+M G \xi^{2}\right) \cosh \xi h .
\end{aligned}
$$

Now the first order reflection and transmission coefficients, $R_{1}$ and $T_{1}$, due to normally incident wave, are obtained by letting $\xi \rightarrow \pm \infty$ in the relation (15) and comparing with (9).

To calculate the first order reflection coefficient $R_{1}$, we let $\xi \rightarrow-\infty$ in relation (15). As $\xi \rightarrow-\infty$, the behaviour of $\phi_{1}(x, y)$ can be obtained by rotating the path of the integral involving the term $\bar{V}(-\xi)$ into a contour in the first quadrant so that we must include the residue term at the pole $\xi=k_{0}$. The path of the integral involving the term $\bar{V}(\xi)$ in the relation (15) is rotated into a contour in the fourth quadrant so that the integral involving the term $\bar{V}(\xi)$ does not contribute as $\xi \rightarrow-\infty$. Then comparing the resultant integral value with (9), we obtain the value of $R_{1}$ as

$$
\begin{aligned}
R_{1} & =-\frac{i K}{k_{0} \Delta^{\prime}\left(k_{0}\right)} \bar{V}\left(-k_{0}\right) \\
& =-\frac{i\left(k_{0} A+B G\right)}{\Delta^{\prime}\left(k_{0}\right)} \int_{-\infty}^{\infty} c(x) e^{2 i k_{0} x} d x,
\end{aligned}
$$

where

$$
\begin{aligned}
& A=\left(k_{0}+M k_{0}^{3}\right) \cosh k_{0} h-K \sinh k_{0} h, \\
& B=\left(k_{0}+M k_{0}^{3}\right) \sinh k_{0} h-K \cosh k_{0} h,
\end{aligned}
$$

and $\triangle\left(k_{0}\right)$ is given in the relation (16) with $\triangle^{\prime}$ denoting the derivative of $\triangle$ with respect to $\xi$.

Similarly, to calculate the first order transmission coefficient $T_{1}$, we take $\xi \rightarrow \infty$ in relation (15). As $\xi \rightarrow \infty$, the behaviour of $\phi_{1}(x, y)$ can be obtained by rotating the path of the integral involving the term $\bar{V}(\xi)$ into a contour in the first quadrant so that we must include the residue term at the pole $\xi=k_{0}$. The path of the integral involving the term $\bar{V}(-\xi)$ in (15) is rotated into a contour in the fourth quadrant so that the integral involving the term $\bar{V}(-\xi)$ does not contribute as $\xi \rightarrow \infty$. Then comparing the resultant integral value with (9), we obtain the value of $T_{1}$ as

$$
T_{1}=-\frac{i\left(-k_{0} A+B G\right)}{\Delta^{\prime}\left(k_{0}\right)} \int_{-\infty}^{\infty} c(x) d x .
$$

These resulting integrals $R_{1}$ and $T_{1}$ can be evaluated once the bottom profile $c(x)$ is known. In the Section 5 , we consider a special form for the shape function $c(x)$. 


\section{Limiting Case}

In the absence of the surface tension and porosity (i.e., $M=$ $0, G=0$ ), the reflection and transmission coefficients in relations (17) and (19) agree with the ones obtained by Miles [4], Davies and Heathershaw [1], Martha and Bora [3], and Mandal and Basu [2] (for $G=0$, impermeable bed).

In the absence of the surface tension, that is, when $M=0$, the above results coincide exactly with the results of Martha et al. [8].

\section{Special Form of the Bottom Profile}

In 1982 Davies [9] found that an undulating bed has the ability to reflect incident wave energy which has an important implication in the application of coastal protection and in the case of possible ripple growth if the bed is erodible. Here we consider a special form of the shape function $c(x)$ in the form of a patch of sinusoidal ripples as the bottom undulation. The shape function is given by

$$
c(x)= \begin{cases}a \sin (l x+\delta) & L_{1} \leq x \leq L_{2}, \\ 0 & \text { otherwise }\end{cases}
$$

where

$$
L_{1}=\frac{-n \pi-\delta}{l}, \quad L_{2}=\frac{m \pi-\delta}{l}
$$

with $a$ as the amplitude of the sinusoidal ripples, $l$ the wave number of the sinusoidal ripples with an arbitrary phase angle $\delta$, and $m, n$ positive integers. For this case, the reflection coefficient $R_{1}$ and the transmission coefficient $T_{1}$, respectively, are obtained as

$$
\begin{gathered}
R_{1}=-\frac{i\left(k_{0} A+B G\right)}{\Delta^{\prime}\left(k_{0}\right)} \frac{a l}{\left(l^{2}-\left(2 k_{0}\right)^{2}\right)} \\
\times\left[(-1)^{n} e^{2 i k_{0} L_{1}}-(-1)^{m} e^{2 i k_{0} L_{2}}\right], \\
T_{1}=-\frac{i\left(-k_{0} A+B G\right)}{\triangle^{\prime}\left(k_{0}\right)}\left(-\frac{a}{l}\right)\left[(-1)^{m}-(-1)^{n}\right] .
\end{gathered}
$$

In the situation in which there is an integer number of ripple wavelengths in the patch $L_{1} \leq x \leq L_{2}$ such that $m=n$ and $\delta=0$, we find $R_{1}$ and $T_{1}$, respectively, as

$$
\begin{gathered}
R_{1}=-\frac{\left(k_{0} A+B G\right)}{\Delta^{\prime}\left(k_{0}\right)} \frac{2 a l(-1)^{m}}{\left(l^{2}-\left(2 k_{0}\right)^{2}\right)} \sin \left(\frac{2 k_{0} m \pi}{l}\right), \\
T_{1}=0 .
\end{gathered}
$$

Relation (23) illustrates that for a given number of $m$ ripples, the first order reflection coefficient $R_{1}$ is an oscillatory in nature. Furthermore, when the bed wave number is approximately twice the surface wave number (i.e., when $l=2 k_{0}$ ), Bragg-resonance described earlier by Davies [9], Mei [10], and Davies and Heathershaw [1] takes place between the bed forms and the surface waves. The relation (23) becomes of the

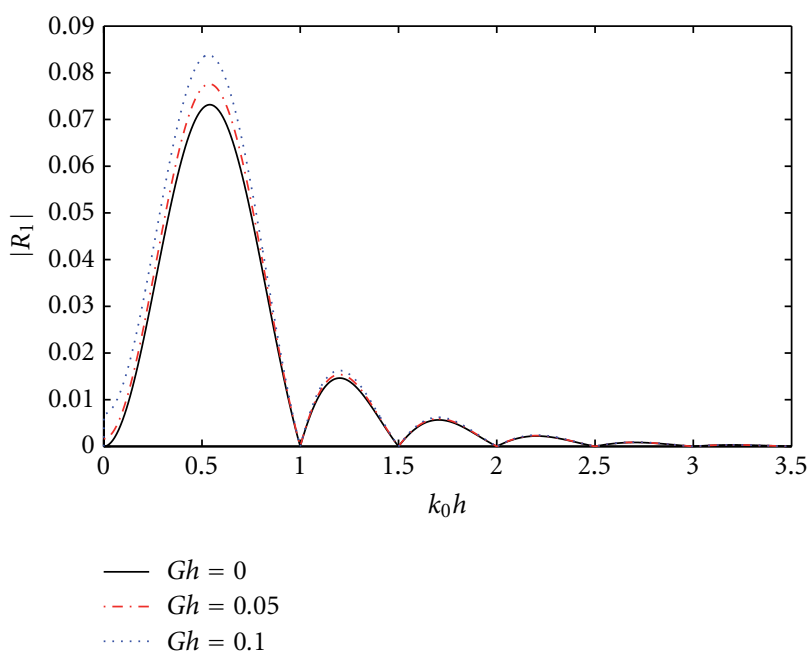

FIGURE 2: Reflection coefficient $\left|R_{1}\right|$ against wave number $k_{0} h$ for $l h=1, a / h=0.1, m=1, M / h^{2}=0$.

form $0 / 0$ when $l=2 k_{0}$ (at resonance), and in such a situation, we employ L'Hôpital's rule to compute the limiting value and find that the reflection coefficient is given by the following expression:

$$
R_{1}=\frac{\left(k_{0} A+B G\right)}{\Delta^{\prime}\left(k_{0}\right)} \frac{a m \pi}{l} .
$$

Thus the reflection coefficient $R_{1}$ becomes a constant multiple of $m$, the number of ripples in the patch. Hence, the reflection coefficient $R_{1}$ increases linearly with $m$. This indicates that relatively few bottom undulation with its wave number equal to approximately twice the surface wave number may give rise to a very substantial reflected wave.

\section{Numerical Results and Discussion}

In this section, the first order reflection coefficient $\left|R_{1}\right|$ as given by (23) is computed numerically for various values of different dimensionless parameter and they are presented graphically.

In Figure 2, the first order reflection coefficient $\left|R_{1}\right|$ is depicted against wave number $k_{0} h$ for different values of the porous effect parameter $G h$ and for the dimensionless parameters $l h=1, a / h=0.1, m=1, M / h^{2}=0$. From this figure, it is clear that $\left|R_{1}\right|$ is oscillatory in nature and the peak value of $\left|R_{1}\right|$ is attained when the wave number of the bottom undulation $l h$ becomes approximately twice the surface wave number $k_{0} h$, which validate the theoretical observation. Also the peak value of the reflection coefficient increases as the value of the porous effect parameter increases.

In Figure 3, the first order reflection coefficient $\left|R_{1}\right|$ is plotted against the wave number $k_{0} h$ for different number of ripples of the bed $(m=1,2,3)$ and for $l h=1, a / h=0.1$, $M / h^{2}=1, G h=0.1$. From this figure, it is concluded that when the number of ripples $m$ of the bed increases, the peak value of $\left|R_{1}\right|$ also increases, which also validate the theoretical 


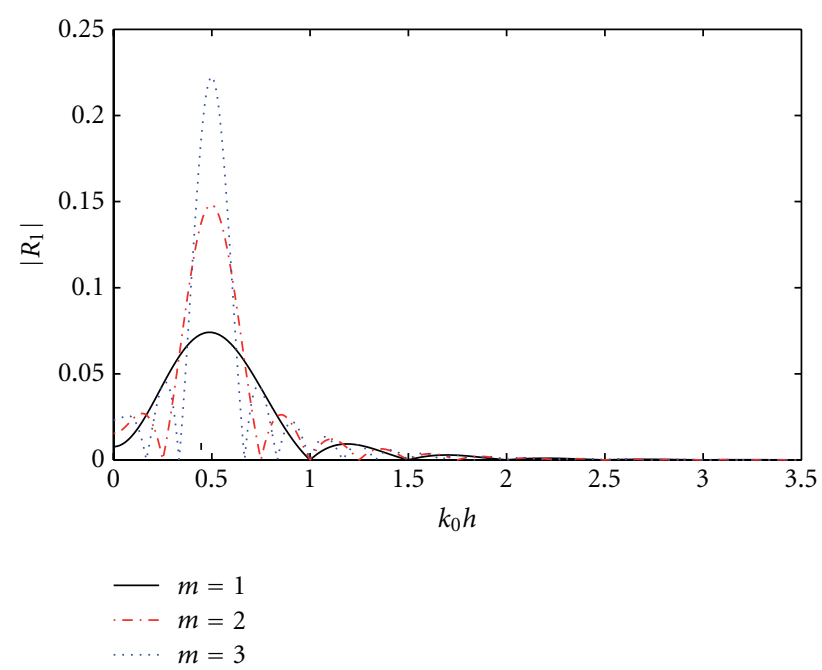

Figure 3: Reflection coefficient $\left|R_{1}\right|$ against wave number $k_{0} h$ for $l h=1, a / h=0.1, M / h^{2}=1, G h=0.1$.

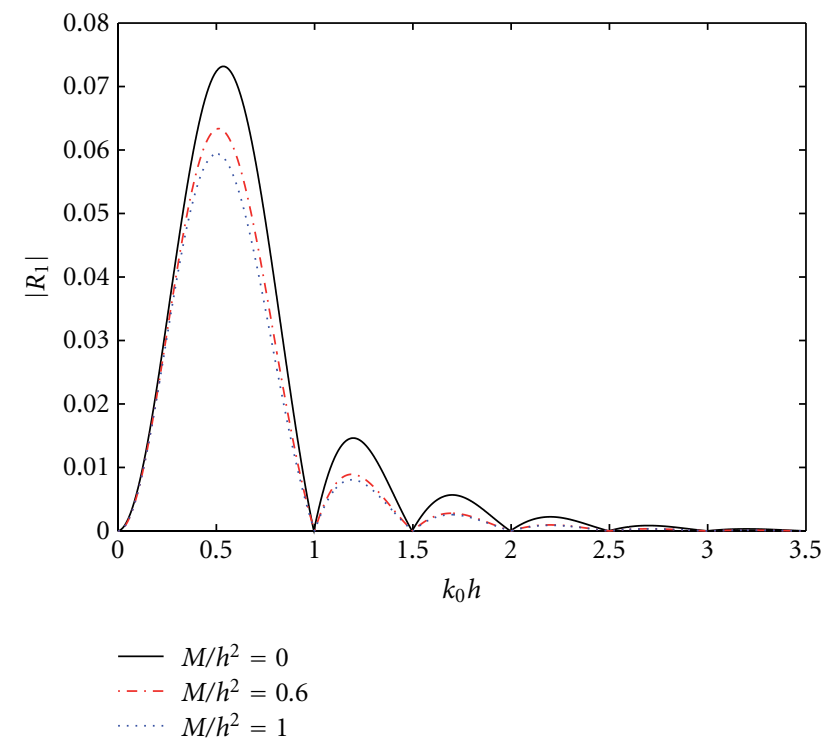

FigURE 4: Reflection coefficient $\left|R_{1}\right|$ against wave number $k_{0} h$ for $l h=1, a / h=0.1, m=1, G h=0$.

observation of (25). It is also clear that its oscillatory nature is more pronounced with the number of zeros of $\left|R_{1}\right|$ increased but the general feature of $\left|R_{1}\right|$ remains the same.

The effect of the surface tension on reflection coefficient $\left|R_{1}\right|$ is shown for $l h=1, a / h=0.1, m=1, G h=0$ in Figure 4 and for the same set of values with $m=2$ in Figure 5. From these figures, it is clear that the value of the first order reflection coefficient decreases as the surface tension increases.

\section{Conclusion}

Scattering of water waves by small undulation of the porous bottom of an ocean in the presence of surface tension is

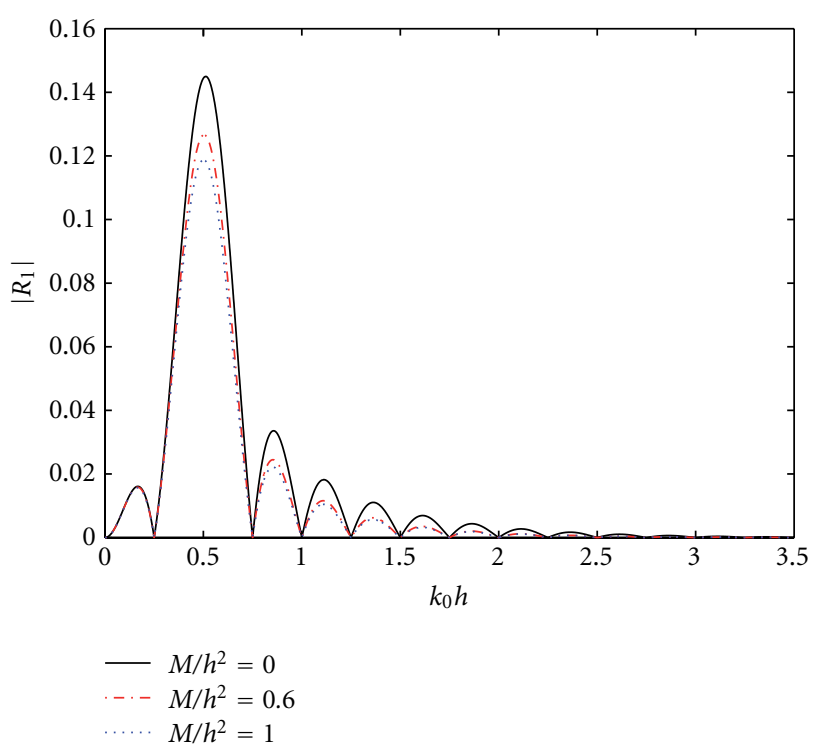

FIGURE 5: Reflection coefficient $\left|R_{1}\right|$ against wave number $k_{0} h$ for $l h=1, a / h=0.1, m=2, G h=0$.

investigated here. Perturbation analysis in conjunction with the Fourier transform technique is used to derive the first order velocity potential, the reflection, and transmission coefficients. The main advantage of this method is that we need to solve relatively easier ordinary differential equation to find the Fourier transform of the velocity potential. The derived results for reflection and transmission coefficients are exactly coincide with the known ones obtained earlier in the case when the bed has no porous effect and when the surface tension at the free surface is neglected. The results for the reflection and transmission coefficients for sinusoidal bottom topography are determined in detail and demonstrated graphically. From the computational results, it is clear that the peak value of reflection coefficient increases when the porosity of the uneven ocean bed increases. Another important conclusion is that the value of the reflection coefficient is found to be decreasing with the surface tension at the free surface. The analysis of the present work which is developed mainly for two-dimensional problems is also applicable to problems for the case of oblique incidence (three dimensions), with appropriate assumptions and modifications and this will be the subject matter of our future work.

\section{Acknowledgment}

S. Panda is grateful to the Council of Scientific and Industrial Research (CSIR), Government of India, for providing the Junior Research Fellowship for pursuing Ph. D. degree at the Indian Institute of Technology Ropar, India. S. C. Martha also thanks the Indian Institute of Technology Ropar, India, for the support through ISIRD grant and for providing all the necessary facilities. The authors thank Professor Aloknath Chakrabarti, Indian Institute of Science, Bangalore, India, for his valuable suggestions and comments during the preparation of the paper. 


\section{References}

[1] A. G. Davies and A. D. Heathershaw, "Surface-wave propagation over sinusoidally varying topography," Journal of Fluid Mechanics, vol. 144, pp. 419-443, 1984.

[2] B. N. Mandal and U. Basu, "A note on water-wave diffraction by a cylindrical deformation of the bottom in the presence of surface tension," Archives of Mechanics, vol. 42, pp. 723-727, 1990.

[3] S. C. Martha and S. N. Bora, "Reflection and transmission coefficients for water wave scattering by a sea-bed with small undulation," ZAMM: Zeitschrift für Angewandte Mathematik und Mechanik, vol. 87, no. 4, pp. 314-321, 2007.

[4] J. W. Miles, "Oblique surface-wave diffraction by a cylindrical obstacle," Dynamics of Atmospheres and Oceans, vol. 6, no. 2, pp. 121-123, 1981.

[5] H. Mase and K. Takeba, "Bragg scattering of waves over porous rippled bed," in Proceedings of the 24th International Conference on Coastal Engineering (ICCE '94), pp. 635-649, ASCE, Kobe, Japan, October 1994.

[6] S. Zhu, "Water waves within a porous medium on an undulating bed," Coastal Engineering, vol. 42, no. 1, pp. 87-101, 2001.

[7] R. Silva, P. Salles, and A. Palacio, "Linear waves propagating over a rapidly varying finite porous bed," Coastal Engineering, vol. 44, no. 3, pp. 239-260, 2002.

[8] S. C. Martha, S. N. Bora, and A. Chakrabarti, "Oblique waterwave scattering by small undulation on a porous sea-bed," Applied Ocean Research, vol. 29, no. 1-2, pp. 86-90, 2007.

[9] A. G. Davies, "The reflection of wave energy by undulations on the seabed," Dynamics of Atmospheres and Oceans, vol. 6, no. 4, pp. 207-232, 1982.

[10] C. C. Mei, "Resonant reflection of surface water waves by periodic sand-bars," Journal of Fluid Mechanics, vol. 152, pp. 315-335, 1985. 

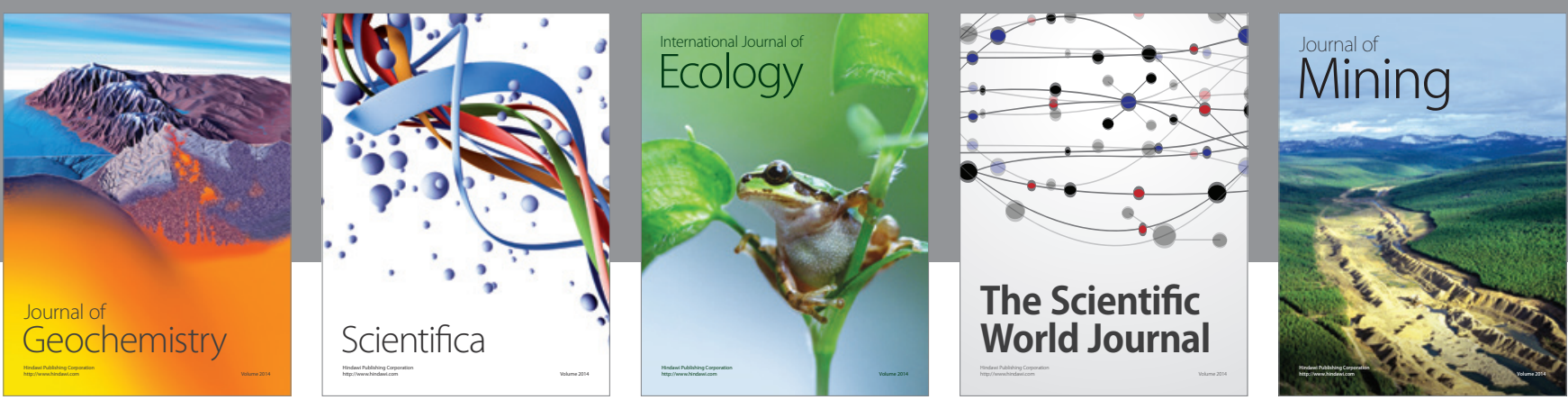

The Scientific World Journal
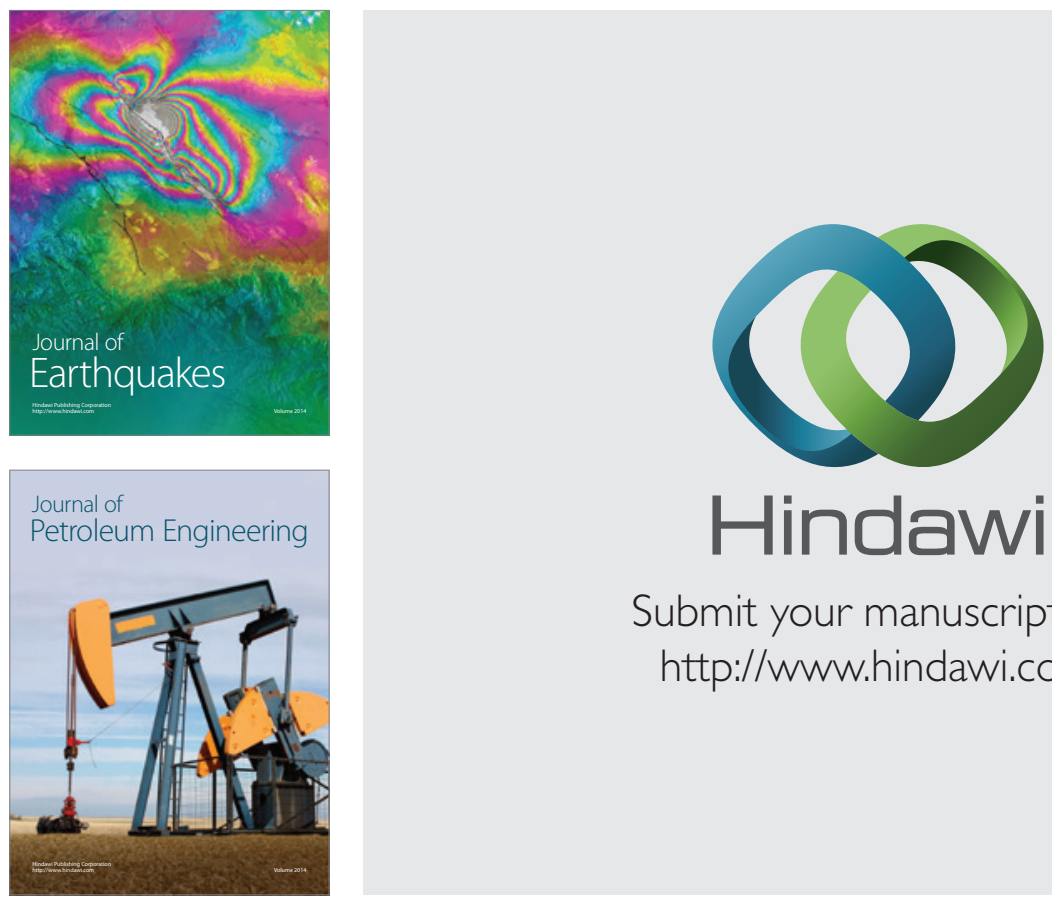

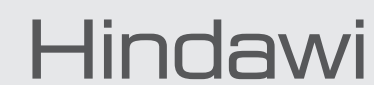

Submit your manuscripts at

http://www.hindawi.com
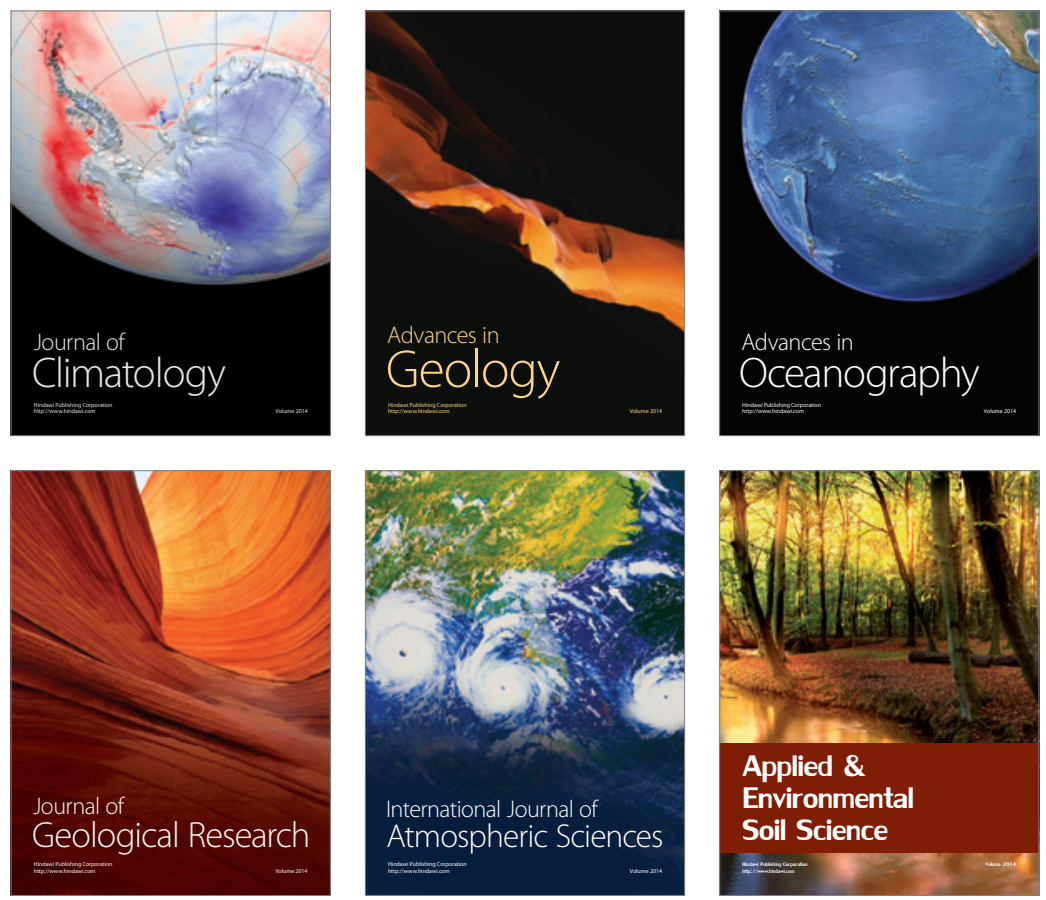
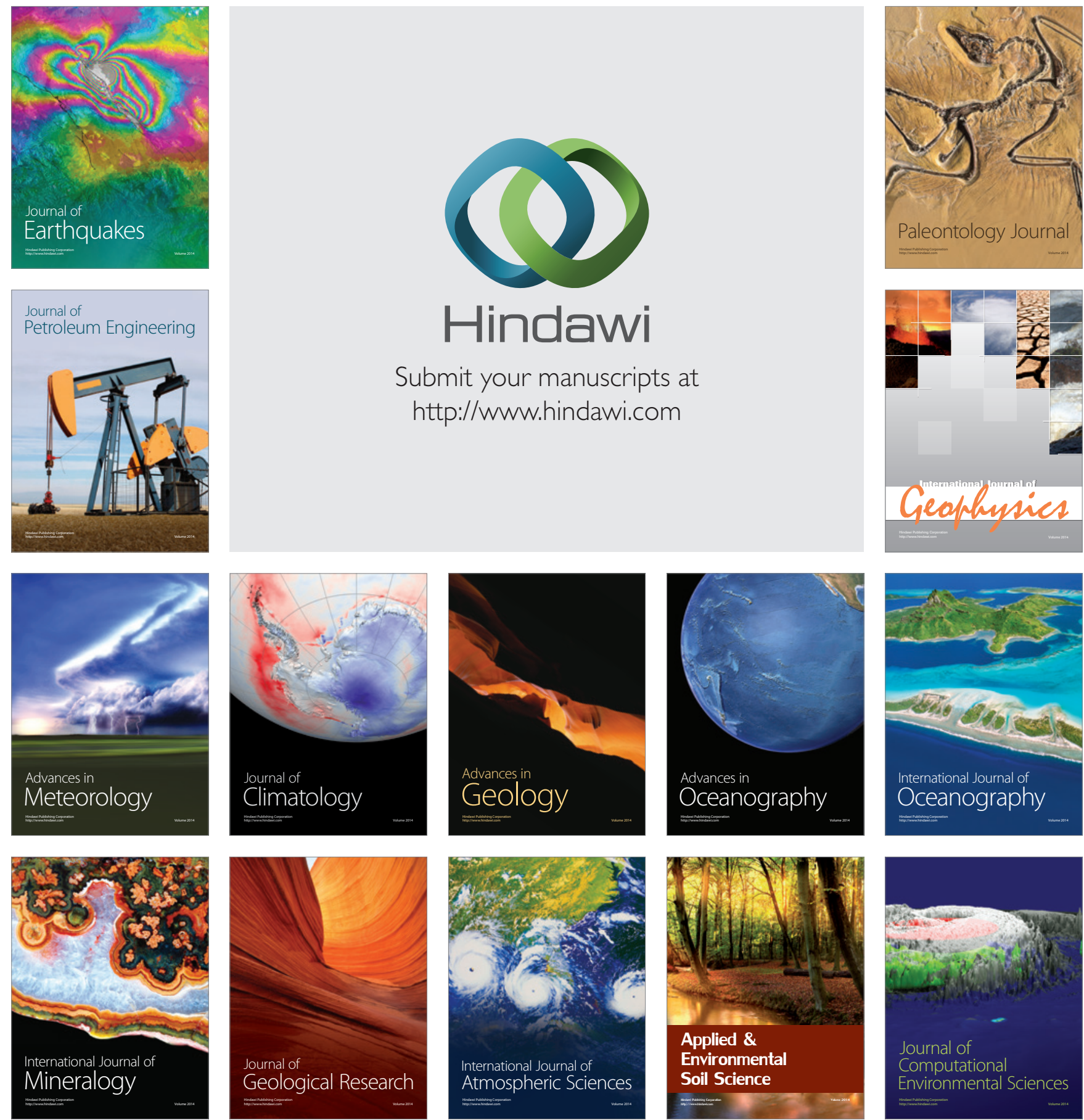